\title{
Epidemiología y manejo del empiema torácico por absceso hepático
}

\author{
Francisco Reyna-Sepúlveda,凶 Marco Hernández-Guedea, Saúl García-Hernández, \\ Jessica Sinsel-Ayala, Edelmiro Pérez-Rodríguez, Gerardo Muñoz-Maldonado
}

Hospital Universitario «Dr. José Eleuterio González», Monterrey, México.

Trabajo recibido: 23-I-2017; aceptado: 15-V-2017

\begin{abstract}
RESUMEN. Antecedentes: El absceso hepático puede ser clasificado en dos categorías: absceso amebiano y absceso piógeno. Sin embargo, la causa más frecuente de absceso hepático en todo el mundo es el amebiano. El empiema suele ser una complicación de la neumonía, pero puede surgir de una infección adyacente como el hígado. Objetivo: Determinar la epidemiología y evaluar el manejo de pacientes con empiema por absceso hepático. Material y métodos: Se solicitaron al Hospital Universitario «Dr. José Eleuterio González» los expedientes correspondientes al período del 2011-2015 con diagnóstico de absceso hepático y empiema. Resultados: Se revisaron 24 pacientes, se reportó diabetes mellitus en 10 (42\%); el síntoma más común fue el dolor abdominal con 18 (75\%), seguido de fiebre y escalofríos con 16 (67\%), disnea en 12 (50\%), con una etiología amebiana en 4 (16\%) de los pacientes y los $20(83 \%)$ restantes piógena. A todos los pacientes se les colocó sonda de toracostomía para drenaje del empiema, 14 (58\%) de los pacientes fueron sometidos a toracoscopia. Se reportaron 2 (8\%) mortalidades. Discusión: La prevalencia de diabetes está asociada con una mayor mortalidad en los abscesos hepáticos. La presentación en la mayoría de los casos fue tardía y ningún paciente acudió con un cuadro clínico de neumonía. Existen reportes de algunos abscesos hepáticos amebianos que causan empiema pleural, pero muy pocos casos de etiología piógena están documentados. Conclusiones: La prevalencia de Klebsiella pneumonie con cuadros clínicos más inespecíficos puede explicar el retraso en el diagnóstico. Reportamos una mortalidad más baja a la previamente establecida.
\end{abstract}

Palabras clave: Absceso hepático, empiema, epidemiología.

ABSTRACT. Background: Liver abscess (LA) can be classified into two categories: amebic (AA) and pyogenic (PA) abscess. However, the most common cause of LA worldwide is amoebic. Empyema is usually a complication of pneumonia, but may arise from an adjacent infection such as the liver. Objective: To determine the epidemiology and to evaluate the management of patients with empyema by LA. Material and methods: We requested the University Hospital «Dr. José Eleuterio González» the records of 2011-2015 with diagnosis of LA and empyema. Results: Twenty-four patients were diagnosed with diabetes mellitus in $10(42 \%)$. The most common symptom was abdominal pain with 18 (75\%) patients, followed by fever and chills with 16 (67\%), dyspnea in $12(50 \%)$, with amoebic etiology in $4(16 \%)$ patients and the remaining 20 $(83 \%)$ was pyogenic. In all patients a thoracostomy tube for empyema drainage was placed, 14 (58\%) of patients underwent thoracoscopy and two (8\%) mortalities were reported. Discussion: The prevalence of diabetes is associated with a higher mortality in LA. The presentation of most of the cases was late and no patient came with a clinical picture of pneumonia. There are reports of some amoebic LA that cause pleural empyema but very few cases of pyogenic etiology have been documented. Conclusions: The prevalence of Klebsiella pneumoniae with more nonspecific clinical features may explain the delay in diagnosis. We report a lower mortality than previously established.

Key words: Empyema, liver abscess, epidemiology.

\section{ANTECEDENTES}

La incidencia y los tipos de abscesos hepáticos varían de un país a otro. El absceso hepático puede ser causado por infección bacteriana, protozoos $u$ hongos. Se clasifica en dos categorías: amebiano y absceso hepático piogénico. En los países desarrollados, el 75\% de los abscesos hepáticos son piógenos. Sin embargo, la causa más frecuente de ellos alrededor del mundo es el absceso hepático amebiano..$^{1-3}$ La fuente de infec- ción puede originarse por el sistema biliar, portal, por vía hematógena y la extensión directa de los órganos adyacentes o el traumatismo penetrante. ${ }^{4,5}$ Alrededor del $15 \%$ de los casos son de etiologías desconocidas.

El absceso hepático piógeno se diagnostica con base en signos y síntomas clínicos, resultados de laboratorio y exámenes radiológicos. Los síntomas que se encuentran comúnmente en el absceso piógeno son fiebre, dolor abdominal en el cuadrante superior derecho, disminución del apetito y pérdida de peso. ${ }^{6}$ 
En el absceso hepático amebiano, más del 95\% de la prueba serológica dará un resultado positivo. La ecografía abdominal puede detectar con precisión el absceso hepático; ${ }^{7,8}$ sin embargo, la tomografía axial computada (TAC) puede ser más precisa con una sensibilidad cercana al $99 \% .^{9,10}$

El metronidazol se administra para superar la infección anaerobia y la amebiasis. Si se ha confirmado el diagnóstico de absceso amebiano se puede continuar con el tratamiento con metronidazol. Si se revela probablemente debido a abscesos piógenos, el tratamiento con antibióticos debe administrarse de acuerdo con la respuesta clínica y el resultado del cultivo de sangre o de líquido de absceso. Los antibióticos parenterales deben administrarse por lo menos durante 48 horas después de que no exista fiebre y leucocitosis y continúe con antibióticos orales. ${ }^{11}$

No todos los abscesos hepáticos deben ser drenados, sólo los grandes que tienen más de $5 \mathrm{~cm}$ de diámetro. Casi siempre el absceso de tamaño pequeño sólo se resolverá con la administración de antibióticos. La intervención quirúrgica es necesaria si hay fracaso del tratamiento o a la punción percutánea. Las complicaciones del absceso hepático son la sepsis, el empiema como extensión directa o la rotura del absceso en la cavidad pleural, la peritonitis debida a la rotura del absceso y el derrame pleuropericárdico.

El empiema suele ser una complicación de la neumonía, pero puede surgir de una infección adyacente que afecta a la orofaringe, el esófago, el mediastino o un tejido subdiafragmático, como el hígado. ${ }^{12}$ La afección pleural o pulmonar del absceso hepático amebiano o piógeno ocurre raramente, esto se desarrolla cuando un absceso del lóbulo derecho afecta por contigüidad el diafragma y produce un empiema o fístula broncopleural. Esta afectación está asociada con dolor en el pecho inferior derecho, por lo común acompañado de tos persistente..$^{13}$

El absceso hepático piógeno tiene mal pronóstico si se sabe que es múltiple, si el paciente tiene otras comorbilidades o el diagnóstico se demora. La mortalidad anteriormente de los nuevos tratamientos era de hasta $77 \% .{ }^{14}$ Hoy, la mortalidad de los abscesos amebianos es del $1-3 \%,{ }^{15}$ de los abscesos piógenos es del $10 \%{ }^{16}$

\section{Objetivo}

Determinar la epidemiología y evaluar el manejo de pacientes con empiema por absceso hepático.

\section{MATERIAL Y MÉTODOS}

Se solicitaron al Departamento de Estadística del Hospital Universitario «Dr. José Eleuterio González» los expedientes de los pacientes mayores de 18 años con diagnóstico de absceso hepático y empiema del 2011-2015. El código CIE-10 utilizado fue K75.0 y J86.0. El estudio tiene un diseño retrospectivo. El criterio de exclusión fue la ausencia del expediente.

Se determinaron diferentes variables como los datos generales del paciente, antecedentes personales patológicos y no patológicos, sintomatología, signos vitales, exámenes de laboratorio, cultivos, antibioticoterapia, tratamiento y se valoró su seguimiento hasta el egreso. El diagnóstico de empiema fue realizado por el Departamento de Neumología. Para el análisis descriptivo se utilizó SPSS versión 22 (IBM, Armonk, NY, USA).

\section{RESULTADOS}

Se obtuvo un total de 25 pacientes, se descartó uno por tener el expediente incompleto. Se revisaron 24 pacientes, de los cuales $10(42 \%)$ fueron del sexo masculino y $14(58 \%)$ del sexo femenino, con una edad promedio de 33.9 años. Dentro de sus antecedentes personales patológicos se reportó diabetes mellitus en 10 (42\%) y $2(8 \%)$ pacientes con hipertensión arterial. En cuanto al tiempo de hospitalización fue de $20 \pm 11$ días de internamiento y 14 (58\%) de los pacientes permanecieron más de dos semanas.

La duración de los síntomas fue de $33 \pm 50$ días. El síntoma más común fue dolor abdominal con 18 (75\%) pacientes, seguido de fiebre y escalofríos con $16(67 \%)$, disnea en 12 (50\%), náusea o vómito en $10(42 \%)$, ictericia en $8(33 \%)$ y pérdida de peso en $2(8 \%)$. Los signos vitales y los resultados de laboratorio se muestran en la tabla 1 . Se obtuvo seroameba positiva en $4(16 \%)$ de los pacientes, los $20(83 \%)$ restantes correspondieron a etiología piógena. En cuanto a los cultivos de los abscesos se obtuvieron 14 (58\%) positivos: 5 Klebsiella pneumonie; 4 Estreptococcus spp; 3 Escherichia coli; y 2 Enteroccocus faecalis.

Con respecto a los estudios de imagen el tamaño promedio fue de $4.4 \pm 5.2$. En la localización, 18 (75\%) de los abscesos ocurrieron en el lóbulo derecho y 6 (25\%) en ambos lóbulos. Con 20 (83\%) pacientes con un solo absceso y el resto con dos o más abscesos. No se reportó pared engrosada, septos, neumobilia o rotura del absceso en ninguno de estos casos.

El tratamiento y el posoperatorio se describen en la tabla 2. A todos los pacientes se les colocó sonda de toracostomía para drenaje del empiema (figura 1), reportando $24(100 \%)$ de empiema localizado del lado derecho y 4 (16\%) ambos lados. De los 14 (58\%) pacientes sometidos a toracoscopia, $12(50 \%)$ fueron 
egresados sin sonda pleural. Los restantes 10 pacientes fueron egresados con sonda pleural. Se reportaron 2 (8\%) mortalidades.

Tabla 1. Signos vitales y exámenes de laboratorio en pacientes con empiema por absceso hepático.

\begin{tabular}{|c|c|}
\hline & n (\%) \\
\hline \multicolumn{2}{|l|}{ Signos vitales } \\
\hline PA sistólica & $109.5 \pm 19$ \\
\hline PA sistólica $<90$ mmHg & $12(50)$ \\
\hline $\mathrm{FC}$ & $104 \pm 18$ \\
\hline $\mathrm{FC}>99$ & $12(50)$ \\
\hline FR & $22 \pm 8.7$ \\
\hline $\mathrm{FR}>20 \mathrm{rpm}$ & $12(50)$ \\
\hline Saturación $\% \mathrm{O}_{2}$ & $97 \pm 1.6$ \\
\hline Saturación $\% \mathrm{O}_{2}^{2}<94 \%$ & 4 \\
\hline Temperatura & $37.6 \pm 0.5$ \\
\hline Temperatura $>37.9^{\circ} \mathrm{C}$ & $6(25)$ \\
\hline IMC & $28.4 \pm 3.9$ \\
\hline IMC > 29 & $6(25)$ \\
\hline \multicolumn{2}{|l|}{ Laboratorio } \\
\hline HGB g/dL & $11.2 \pm 1.6$ \\
\hline $\mathrm{HGB}<10 \mathrm{~g} / \mathrm{dL}$ & $12(50)$ \\
\hline Leucocitos K/uL & $13.6 \pm 5.2$ \\
\hline Leucocitos > $14 \mathrm{~K} / \mathrm{uL}$ & $12(50)$ \\
\hline Albúmina g/dL & $2.3 \pm 0.4$ \\
\hline Albúmina $<3.0 \mathrm{~g} / \mathrm{dL}$ & $24(100)$ \\
\hline BT mg/dL & $1.1 \pm 0.7$ \\
\hline $\mathrm{BT}>2.0 \mathrm{mg} / \mathrm{dL}$ & $2(8)$ \\
\hline AST UI/L & $60 \pm 68.9$ \\
\hline AST 2x UI/L & $4(17)$ \\
\hline ALT UI/L & $43 \pm 63$ \\
\hline ALT 2x UI/L & $2(8)$ \\
\hline FA UI/L & $186 \pm 115$ \\
\hline $\mathrm{FA}>147 \mathrm{UI} / \mathrm{L}$ & $10(42)$ \\
\hline
\end{tabular}

APNP = Antecedentes personales no patológicos; PA = Presión arterial; FC = Frecuencia cardíaca; FR = Frecuencia respiratoria; IMC = Índice de masa corporal; HGB = Hemoglobina; AST = Aspartato aminotransferasa; $\mathrm{ALT}=$ Alanino aminotransferasa; $\mathrm{FA}=$ Fosfatasa alcalina; $\mathrm{ABT}=$ Antibioticoterapia.

Tabla 2. Tratamiento y evolución posoperatoria de pacientes con empiema por absceso hepático.

\begin{tabular}{lc}
\hline & $\mathrm{n}(\%)$ \\
\hline Tratamiento & \\
$\quad$ Conservador/punción & $22(92)$ \\
Quirúrgico & $2(8)$ \\
Segundo procedimiento & $12(50)$ \\
Toracoscopia & $14(58)$ \\
Terapia intensiva & $2(8)$ \\
Recurrencia & $4(17)$ \\
Mortalidad & $2(8)$ \\
\hline
\end{tabular}

\section{DISCUSIÓN}

La prevalencia de diabetes está asociada con una mayor mortalidad en los abscesos hepáticos. La prevalencia en México es de $9.2 \%$ en adultos y en nuestro estudio fue de $42 \%$. La mayoría de los pacientes duraron un largo período de internamiento por los múltiples procedimientos realizados, además de la necesidad de cumplir con esquemas completos de antibioticoterapia. La prevalencia del absceso pleural en el caso de absceso hepático reportada es de $4.98 \% .^{15}$

En México el manejo actual ha cambiado. En una revisión de absceso hepático y empiema toracis realizada por Medina-Morales et al. ${ }^{17}$ en 1997, el manejo quirúrgico del empiema era sonda pleural, requiriendo decorticación o resección pulmonar sólo el $8.4 \%$. Actualmente, y a diferencia de nuestro estudio, el $50 \%$ fue de abordaje toracoscópico.

La presentación de la mayoría de los casos fue tardía, los abscesos hepáticos amebianos son más grandes, se presentan con mayores complicaciones y tienen menor respuesta al tratamiento, ${ }^{18}$ probablemente asociada a la falta de atención médica oportuna de la población afectada. En nuestra serie, el promedio de atención de la sintomatología fue de 33 días, adicionado al hecho de que la mitad de los pacientes acudieron hasta presentar disnea asociada a datos de inestabilidad hemodinámica a su llegada al área de Urgencias. El diagnóstico temprano es esencial, el retraso de éste se relaciona con un incremento en la mortalidad..$^{19,20}$

En la disnea, a pesar de que la mitad de los pacientes la presentaban, sólo cuatro de ellos llegaron con

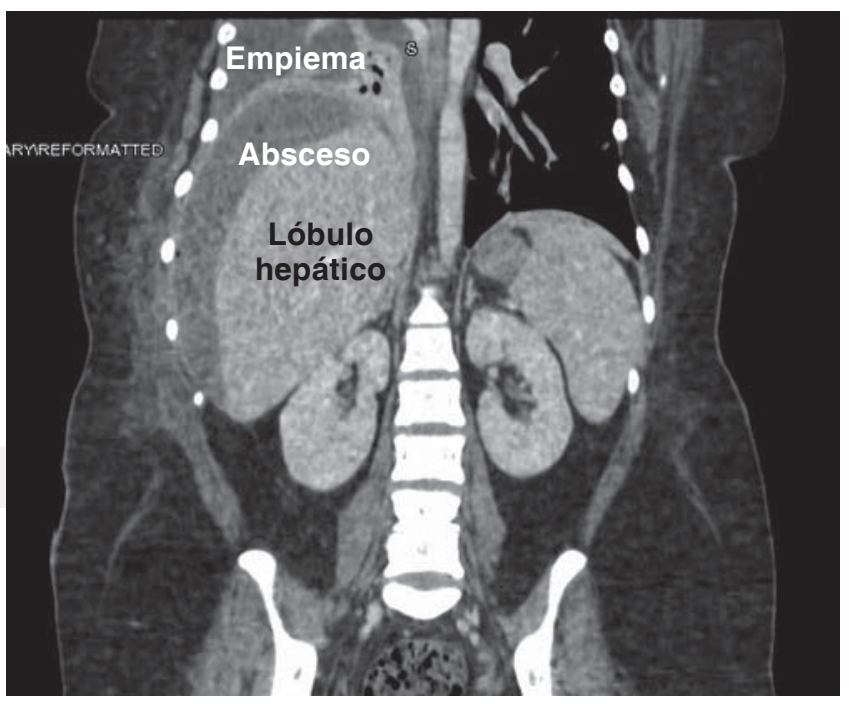

Figura 1. Tomografía axial computada de paciente de empiema por absceso hepático. 
saturaciones de $\mathrm{O}_{2}$ inferiores a $94 \%$, lo que se traduce en quizás dolor pleurítico, presentado al empiema de base que manifestaban. Ningún paciente acudió con un cuadro clínico de neumonía. En los exámenes de laboratorio la mitad presentó anemia normocítica normocrómica y leucocitosis; también, BT, AST y ALT sin cambios importantes, sólo la FA resultó elevada en $42 \%$ de los casos. Por imagen ningún absceso presentó septos, pared engrosada o aire.

La Klebsiella pneumonie es un patógeno que por lo común sobreinfecta el empiema amebiano, se ha aislado cada vez con más frecuencia en este tipo de formación de abscesos. En México es el patógeno más comúnmente aislado, ${ }^{21}$ siendo antes Escherichia coli. La prevalencia de Klebsiella pneumonie con cuadros clínicos más inespecíficios ${ }^{22}$ puede explicar el retraso en el diagnóstico y las etapas más avanzadas de nuestros pacientes. No todos los abscesos resultaron en cultivos positivos, esto se debe a la antibioticoterapia previa. Además, la seroameba negativa puede deberse a que el absceso hepático amebiano agudo puede tener un resultado falso negativo. ${ }^{23}$

Existen reportes de algunos abscesos hepáticos amebianos que causan empiema pleural, pero muy pocos casos de etiología piógena están documentados. ${ }^{24,25}$ Los mecanismos teóricos de la diseminación torácica incluyen: la rotura directa de un absceso hepático a través del diafragma; propagación linfática a través del diafragma; diseminación hematógena de trofozoítos; o la inhalación de quistes de Entamoeba histolytica. ${ }^{26}$

A todos los pacientes se les colocó sonda pleural por empiema previo diagnóstico, ${ }^{27}$ al no verse mejoría clínica y radiográfica se solicitó TAC y se programó para toracoscopia y decorticación. Se retiró la sonda a su egreso sin mayores complicaciones, sólo dos casos se egresaron con la sonda abierta. Retrasar el manejo inicial complica las opciones terapéuticas. La toracoscopia ha demostrado ser útil en todas las fases del empiema, al presentar menor dolor, menor complicación posoperatoria y menor morbimortalidad que la cirugía abierta. ${ }^{28}$

En el manejo de los abscesos hepáticos, la mayoría de los pacientes respondió favorablemente al manejo conservador o con punción percutánea debido a que todos eran abscesos únicos, no loculados. Sólo a dos pacientes se les dio manejo por laparoscopia, en el cual se documentó la ruptura transdiafragmática del absceso. Ésta es una complicación aún más infrecuente del absceso hepático y ha recibido poca atención en la literatura. Hace cuarenta años esta complicación se asoció con una tasa de mortalidad del $77 \%{ }^{29}$

La mortalidad que reportamos (8\%) fue de dos pacientes de edad avanzada con diabetes mellitus y sepsis, la que se asoció a una neumonía intrahospitalaria.
La mortalidad en pacientes de empiema con absceso hepático ha cambiado con las nuevas opciones terapéuticas y antibioticoterapia disponible.

\section{Limitaciones del estudio}

Al ser un estudio retrospectivo una de las principales limitantes fue no poder discernir sobre el juicio clínico del cirujano para la decisión de dar o no un manejo conservador, mínimamente invasivo o por cirugía abierta.

\section{CONCLUSIONES}

La presentación de la mayoría de los casos fue tardía, la prevalencia de Klebsiella pneumonie con cuadros clínicos más inespecíficos puede explicar el retraso en el diagnóstico. El manejo de los abscesos hepáticos sigue siendo antibioticoterapia adicionado al tratamiento conservador y con punción percutánea en su mayoría, dejando como última opción la cirugía. Para el manejo del empiema se utiliza la sonda de toracostomía y la toracoscopia, que ha demostrado ser útil en todas las fases del empiema. Reportamos una mortalidad más baja a la previamente establecida.

\section{REFERENCIAS}

1. Valenzuela O, Morán P, Gómez A, et al. Epidemiology of amoebic liver abscess in Mexico: the case of Sonora. Ann Trop Med Parasitol 2007;101(6):533-538.

2. Okano H, Shiraki $\mathrm{K}$, Inoue $\mathrm{H}$, et al. Clinicopathological analysis of liver abscess in Japan. Int J Mol Med 2002;10(5):627-630.

3. Tamez A, Guillén N, Castorena G. Absceso hepático amibiano múltiple. Rev Asoc Mex Med Crít y Ter Int 2009;23(3):165-172.

4. Zalesnik F, Kasper DL. Intraabdominal infections and abscess. In: Braunwald E, Fauci AS, Kasper DL, Hauser $\mathrm{SL}$, Longo DL, Jameson JL, editors. Harrison's principles of internal medicine. 15th ed. USA: McGraw Hill; 2001. p.829-834.

5. Rodríguez-Herrera R, Carbajal-Rodríguez L, ZarcoRomán J, et al. Absceso hepático amibiano complicado con rotura intraabdominal y torácica. Rev Enfer Infec Pediatr 2010;24(94):64-68.

6. Red SL. Amebiasis and infection with free living amebiasis. In: Braunwald E, Fauci AS, Kasper DL, Hauser SL, Longo DL, Jameson JL, editors. Harrison's principles of internal medicine. 15th ed. USA: McGraw Hill; 2001.p.1200-1202.

7. Stain SC, Yellin AE, Donovan AJ, Brien HW. Pyogenic liver abscess. Modern Treatment. Arch Surg 1991;126(8):991996.

8. Liew KV, Lau TC, Ho CH, et al. Pyogenic liver abscess: $A$ tropical centre's experience in management with review of current literature. Singapore Med J 2000;41(10):489-492. 
9. Wong WM, Wong BC, Hui CK, et al. Pyogenic liver abscess: Retrospective analysis of 80 cases over a 10year period. J Gastroenterol Hepatol 2002;17(9):10011007.

10. Mohsen AH, Green ST, Read RC, McKendrick MW. Liver abscess in adult: ten years' experience in a UK centre. QJM 2002;95(12):797-802.

11. Stain SC, Yellin AE, Donovan AJ, Brien HW. Pyogenic liver abscess: Modern Treatment. Arch Surg 1991;126(8):991996.

12. Vardhan MV, Tewari SC, Prasad BNBM, Nikumb SK. Empyema thoracis-study of present day clinical \& etiological profile and management techniques. Ind J Tub 1998; 45(3):155-160.

13. Sosa-Juárez A, García-Sancho C, Sánchez-Hernández JD, et al. Epidemiologia del derrame pleural en el INER, 2011-2012. Neumol Cir Torax 2013;72(2):136-141.

14. Wong WM, Wong BC, Hui CK, et al. Pyogenic liver abscess: retrospective analysis of 80 cases over a 10-year period. J Gastroenterol Hepatol 2002;17(9):1001-1007.

15. Stanley SL Jr. Amoebiasis. Lancet 2003;361(9362): 10251034.

16. Chen $\mathrm{W}$, Chen $\mathrm{CH}$, Chiu $\mathrm{KL}$, et al. Clinical outcome and prognostic factors of patients with pyogenic liver abscess requiring intensive care. Crit Care Med 2008;36(4):11841188. doi: 10.1097/CCM.0b013e31816a0a06.

17. Medina-Morales F, Echegoyen-Carmona R, ChavarríaGarcés J, Flores-Vergara H. El absceso hepático y sus complicaciones torácicas e la Meseta Central de México. Rev Inst Nal Enf Resp Mex 1997;10(3):168-174.

18. Puebla-Clark JG, Alday-Noriega MC, Peña-Ríos DH. Particularidades del absceso hepático amebiano en México: revisión de una cohorte de pacientes del Hospital General del Estado de Sonora. Med Int Mex 2012;28(5):440-445.

19. Shimada H, Ohta S, Maehara M, Katayama K, Note M, Nakagawara G. Diagnostic and therapeutic strategies of pyogenic liver abscess. Int Surg 1993;78(1):40-45.

20. Lee KT, Wong SR, Sheen PC. Pyogenic liver abscess: an audit of 10 years' experience and analysis of risk factors. Dig Surg 2001;18(6):459-465. discussion 465-466.
21. García-Álvarez J, Rebollar-González RC, Trejo-Téllez R, Sánchez-Conde RM. Abscesos hepáticos piógenos. Rev Hosp Jua Mex 2011;78(3):156-163.

22. Carrillo-Esper $R$, Soto-Hernández $J L$, Peña-Pérez $C A$, Carrillo-Córdova LD, Carrillo-Córdova CA, CarrilloCórdova DM. Síndrome de absceso hepático secundario a Klebsiella pneumoniae hipermucoviscosa con involucro pulmonar. Gac Med Mex 2013;149(1):102-107.

23. Sherlock S, Dooley J. Diseases of the liver and biliary system. 11th ed. London: Blackwell Publ; 2002.p.495497.

24. Cicero-Sabido R, Páramo-Arroyo RF, Navarro-Reynoso FP, Pimentel-Ugarte L. Procedimientos quirúrgicos en 156 casos de derrame pleural. Resultados inmediatos. Cir Ciruj 2006;74(6):409-414.

25. Loulergue $\mathrm{P}$, Mir O. Pleural empyema secondary to amebic liver abscess. Int J Infect Dis 2009;13(3):e135136. doi: 10.1016/j.ijid.2008.07.017.

26. Lyche KD, Jensen WA. Pleuropulmonary amebiasis. Semin Respir Infect 1997;12(2):106-112.

27. Villena-Garrido V, Cases-Viedma E, Fernández-Villar A, et al. Normativa sobre el diagnóstico y tratamiento del derrame pleural. Actualización. Arch Bronconeumol 2014;50(6):235-249. doi: 10.1016/j.arbres.2014.01.016

28. Tong BC, Hanna J, Toloza EM, et al. Outcomes of video-assisted thoracoscopic decortication. Ann Thorac Surg 2010;89(1):220-225. doi: 10.1016/j. athoracsur.2009.09.021.

29. Ochsner A, DeBakey ME. Pleuropulmonary complications of amoebiasis. J Thorac Surg 1936;5:225-258.

\section{$\triangle$ Correspondencia:}

Dr. Gerardo Muñoz-Maldonado

Departamento de Cirugía General, Hospital Universitario «Dr. José Eleuterio González». Francisco I. Madero Pte. y Av. Gonzalitos s/n Col. Mitras, Monterrey, Nuevo León, México. Teléfono: (81) 83467800

Correo electrónico: publications.uanl@gmail.com

Los autores declaran no tener conflicto de intereses 